\title{
Epistemología del paisaje arquitectónico. La generación de conocimiento patrimonial desde la arquitectura
}

\author{
Epistemology of the architectural landscape. The \\ formulation of heritage knowledge from architecture
}

\section{Resumen}

Aurora Villalobos Gómez* aurora.villalobos@juntadeandalucia.es

*Consejería de Cultura y Patrimonio Histórico, Junta de Andalucía nvestigar los procesos de generación de conocimiento patrimonial desde la arquitectura implica, en primer lugar, reconocer las cuestiones de común Linterés en arquitectura y patrimonio para, en segundo lugar, plantear la estrategia para su abordaje. Se propone una investigación epistemológica desde lo general a lo concreto, que delimite el marco conceptual del patrimonio arquitectónico, identifique las posibles líneas de investigación en arquitectura e ilustre su correspondencia en la práctica patrimonial con diversos casos representativos de nuestro contexto cultural. Se presentan el espacio, la arquitectura, el patrimonio y el paisaje como una secuencia de conceptos que definen la relación de las personas con su medio a través de una valoración de tipo cultural. A su vez, se describen y ejemplifican una serie de acciones de creación, investigación y planificación susceptibles de generar dicho conocimiento patrimonial desde la arquitectura. Se concluye con un balance de los resultados y el planteamiento de nuevas líneas de trabajo.

Palabras clave: espacio; arquitectura; patrimonio; paisaje; investigar.

\section{Abstract:}

Investigating the processes of generation of heritage knowledge from architecture implies, firstly, recognizing the issues of common interest in architecture and heritage, and secondly, proposing the strategy for their approach. An epistemological research is proposed from the general to the concrete, which delimits the conceptual framework of the architectural heritage, identifies the possible lines of research in architecture and illustrates its correspondence in the heritage practice with various representative cases of our cultural context. Space, architecture, heritage and landscape are presented as a sequence of concepts that define the relationship of people with their environment through a cultural assessment. In turn, a series of creation, research and planning actions capable of generating heritage knowledge from architecture are described and exemplified. It concludes with a balance of the results and the proposal of new lines of work.

Keywords: : space; architecture; heritage; landscape; research. 


\section{Introducción}

Desde la primera distinción platónica entre 'episteme' (conocimiento) y 'doxa' (opinión), se ha configurado la 'epistemología' como la ciencia que aborda la teoría del conocimiento verdadero (Ferrater Mora, 1965); dicho de otro modo, la relación entre el objeto y el sujeto en el acto del conocimiento (Lalande, 1966). Dado que investiga los fundamentos y métodos de creación del conocimiento, en este artículo se usa el término 'epistemología del paisaje arquitectónico' como el estudio de las formas (el cómo) de generación del conocimiento patrimonial en su paradigma actual del paisaje cultural (el objeto) desde la arquitectura (el sujeto).

Es un hecho que la arquitectura es una disciplina propositiva que construye nuevos espacios, susceptibles de ser considerados patrimonio en función de una valoración de tipo cultural. Asimismo, cuando se interviene sobre una arquitectura heredada, el proyecto arquitectónico puede aportar nuevos significados que ayuden a desvelar los valores culturales de las preexistencias. Por lo tanto, en el devenir de la arquitectura como patrimonio arquitectónico se da un proceso de re-conocimiento que nos interesa afrontar siendo conscientes de su complejidad.

Este planteamiento epistemológico de la investigación implica, como punto de partida desde lo general, definir conceptos interrelacionados en este campo de conocimiento interdisciplinar e identificar acciones de generación de conocimiento patrimonial para tener como objetivos, hacia lo concreto, seleccionar prácticas arquitectónicas representativas de nuestro contexto cultural que sirvan de referencia para la creación de paisaje cultural y abrir nuevas líneas de investigación.

\section{Metodología}

La metodología es el instrumento que dota de sistematicidad una ciencia. Resulta más evidente en disciplinas técnicas, donde los procedimientos son reproducibles mediante experimentos empíricos, pero no es menos necesaria en otras disciplinas que requieren garantizar la calidad del proceso y una evaluación cualitativa de los resultados cuando son varias las posibles soluciones válidas. En patrimonio cultural son muchas las disciplinas que concurren, de manera que resulta de gran ayuda disponer de glosarios comunes, documentos normalizados e indicadores de buenas prácticas.

Es por ello que la primera parte de esta contribución se inicia con una deriva conceptual que establece los postulados teóricos de partida relativos al espacio, la arquitectura, el patrimonio y el paisaje, ordenados de mayor a menor nivel de abstracción. Se parte del espacio por tratarse de la materia base de la arquitectura y se concluye en el paisaje por ser el paradigma contemporáneo del patrimonio cultural.

De cada uno de estos conceptos se presenta un mapa semántico previo que procura poner de manifiesto el marco de complejidad en el que se desenvuelve esta investigación que, ni tiene su punto de partida en este artículo ni pretende concluirla en él. Denotan una toma de conciencia en cuanto al origen disciplinar y las consecuencias patrimoniales de los diversos matices y procuran aportar los fundamentos teóricos para esta epistemología del paisaje arquitectónico. A continuación, se exponen las cuestiones de mayor interés para encuadrar la cuestión y se concluye con una definición representativa del posicionamiento teórico.

En la segunda parte, se desarrollan los resultados de aplicar este enfoque epistemológico a diversas situaciones posibles en la práctica de la arquitectura como generadora de paisajes arquitectónicos.

Se procederá al análisis del cómo se ilustra con experiencias reales de intervención en patrimonio, que se entienden paradigmáticas por la manera en que conservan la materialidad del bien cultural y explicitan sus valores. Entre profundizar en algunos ejemplos o avanzar las claves de diversos casos, se ha preferido la segunda opción para evidenciar la capacidad de la arquitectura para generar conocimiento patrimonial; la necesaria limitación textual de este artículo nos ha movido a optimizar la información, dando prioridad al enfoque respecto al dato concreto. Si bien se ha procurado aportar experiencias recientes (impregnadas de esta nueva intencionalidad patrimonial), es inevitable la mención de otros proyectos modernos más alejados en el tiempo (en los que su contribución cultural sigue siendo actual e inspiradora). De igual modo sucede con las referencias bibliográficas.

\section{Deriva conceptual}

\subsection{Espacio}

Estamos acostumbrados a pensar que la arquitectura es lo construido cuando lo cierto es que no habitamos las paredes, las bóvedas o las ventanas, sino el espacio que media entre ellas, que nos permite desplazarnos, asomarnos, descansar o sentirnos protegidos. Esta idea preconcebida deviene de una manera tradicional de concebir la historia de la arquitectura como la secuencia de una serie de estilos arquitectónicos, en vez de un análisis de las distintas maneras de configurar el espacio (Figura 1).

El historiador Sigfried Giedion supo plantear una historia de las edades del espacio en arquitectura; caracterizada primero por el predominio del espacio exterior (sean los volúmenes de las pirámides o los pedestales que elevan los templos griegos), luego por el predominio del espacio interior (conseguido con los grandes ventanales en la bóveda romana, las tracerías góticas o las escalinatas barrocas), y actualmente por la desaparición de los límites interior-exterior (a partir de la Revolución Industrial, con la diferenciación entre sistema portante y cerramiento). 
espacio funcional

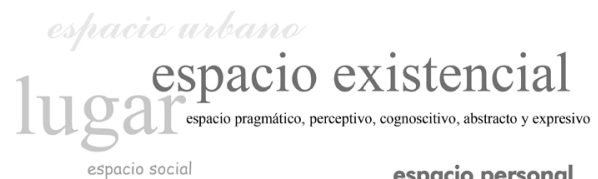

espacio socia

espacio personal

espacio mental

ESPACT0 FÍSIC0 espacio perceptible

$$
\begin{array}{cl}
\text { espacio-direccionat } & \begin{array}{l}
\text { espacio positivo } \\
\text { espacio negativo }
\end{array} \\
\text { no-lugar } &
\end{array}
$$

Figura 1: Mapa semántico: Espacio

Fuente: A. Villalobos Gómez(2020)

Sin ser una teoría completa, sin embargo, supo abrir nuevas investigaciones en cuanto a los tipos de espacio y sus cualidades. El espacio físico sería el punto de partida imprescindible como soporte material que permite la percepción y disfrute de todos los demás. A partir de ahí, el espacio mental sería la imagen que cada uno tiene de contexto que le rodea, el espacio personal sería el que cada uno se reserva de manera unilateral respecto a los demás y el espacio social trascendería a la escala de las relaciones de reciprocidad. Estos enfoques y escalas nos influyen en la forma de relacionarnos con dichos espacios y asignarles funciones.

La fenomenología del espacio planteada por el arquitecto Christian Norberg-Schulz lo ordena en una escala creciente de abstracción: espacio pragmático (en el que la persona actúa), perceptivo (aquel tangible por medio de los cinco sentidos aunque no estemos presentes en él), existencial (el que construye la identidad cultural), cognoscitivo (el del conocimiento dado por las diversas disciplinas), expresivo (la imagen del mundo creada por las artes), estético (en el que se desenvuelve la teoría del arte) y el lógico (el de las relaciones abstractas).

Este conjunto de relaciones que empieza a establecer el ser humano con las cosas (interior-exterior, cercanolejano, pequeño-grande, abierto-cerrado, horizontalvertical, curvilíneo-rectilíneo, centralizado-lineal, continuo-discontinuo, estático-dinámico, positivonegativo) convierte el espacio en un 'lugar' o medio ambiente que es reconocido como parte esencial de la existencia de la persona porque va más allá del entorno y aspira a mejorar sus condiciones de vida. No basta que se satisfagan unas necesidades vitales derivadas de su propia finitud humana, sino también trascendentes, que aporten sentido y orden a un mundo de conocimientos y acciones; de lo contrario estaríamos frente a un 'nolugar'. Es por ello que nos quedamos con la definición del espacio arquitectónico como "la concretización del espacio existencial del hombre" (Norberg-Schulz, 1975, p. 12).

\subsection{Arquitectura}

No basta con una arquitectura entendida como e 'arte del espacio' acercándose a esta idea desde un análisis formalista (como geometría que excluye a la persona) o psicológico (como sensaciones que obvian la materialidad de la arquitectura). Hace falta "una teoría

del espacio realmente interpretado como una dimensión de la existencia humana, más que como una dimensión del pensamiento o de la percepción" (Norberg-Schulz, 1975, p.15). La definición del espacio como "lo que hay en el aire, lo que hay entre las cosas" (Navarro, 1999, p. 113) se trasciende a partir de la experiencia vital del habitante, que lo convierte en "un trozo de aire humanizado" (Fisac, citado por González-Calero, 1998, p. 42). Y es que la idea de un espacio a la medida de la persona nos remite necesariamente a la necesidad de la arquitectura en todas sus manifestaciones (Figura 2).

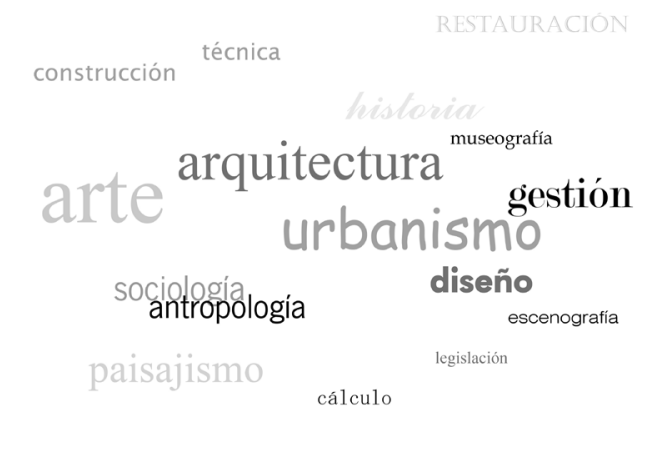

Figura 2: Mapa semántico: Arquitectura Fuente: A. Villalobos Gómez (2020)

Para el filósofo Martin Heidegger "construir es en sí mismo el habitar" (1994, p. 128) ya que no habitamos porque hayamos construido, sino que construimos en la medida en que habitamos. En cierta manera todos tenemos una experiencia del espacio que nos hace sentir creadores de este espacio existencial. El arquitecto es un habitante que se singulariza por su condición propositiva del habitar, es decir, que no solo participa de esos espacios, sino que interviene en su creación: pensar-habitar-construir son así las tres acciones en las que se desglosa su actividad. Ser arquitecto no es solo una profesión sino un modo de ser en el mundo. Por lo tanto, este construir se despliega, por un lado, en el construir que edifica (aedificare), y, por otro, en el que cuida (collere, de donde viene 'cultura'); es decir, lo que hemos venido a llamar 'arquitectura' y 'patrimonio'.)

Esta teoría del espacio existencial tiene su correspondencia en una manera de proyectar que busca la naturaleza de las cosas, teniendo en cuenta la relación entre el carácter del lugar y la actividad propuesta por el hombre. Podemos hablar de unos niveles que estructuran el espacio existencial por escalas: territorial, urbano, residencial, objetual y cósico.

El instrumento efectivo es el 'proyecto', entendido en su sentido más amplio como idea (iniciativa de transformación), documento (síntesis textual y gráfica que permite la ejecución de la idea) y proceso (secuencia de actuaciones que hace realidad lo proyectado). A partir de aquí:

El problema del proyecto se afronta desde el orden; que es aquello que nos permite distinguir la naturaleza de nuestro problema de los espacios que queremos delimitar o abrir en la realidad. A partir de su naturaleza concebimos un espacio donde se percibe su voluntad de ser (Bonaiti, 2002, p.70). 
Para el arquitecto Louis Kahn la naturaleza representa e porqué, el orden es el qué y el proyecto trata del cómo. La naturaleza es aquella ley inmutable que debe ser percibida al traducir la realidad por medio del proyecto, el orden es la regla modificable por el hombre que mira por la presencia de la forma, y "proyecto significa asignar dimensiones y buscar los medios para realizar la forma" (Bonaiti, 2002, p. 83).

Decidir los límites, escala, materialidad... requiere de determinados conocimientos (diseño, arte, construcción, historia, sociología, antropología, geografía y legislación entre otros), y capacidades (analizar, resolver, dirigir, coordinar o planificar), lo que hace de la arquitectura una disciplina propositiva y en sí misma interdisciplinar que, sin dejar de ser generalista, desarrolla diversas especialidades.

\subsection{Patrimonio}

La relación entre arquitectura y patrimonio es evidente desde sus inicios, no solo por la identificación de ambos a través de la idea de 'monumento', sino por la misma idea de 'proyecto'. La comprensión del patrimonio (Figura 3) como objeto vulnerable y de interés para la colectividad ha implicado la necesidad de procurarle, por medio de proyecto, una existencia prolongada para transmitirlo.

\section{hatimonias especiales patrimonio mueble batrimonio históriç patrimonio cultural

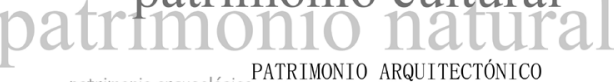 \\ patrimonios emergentes \\ patrimonio etnológico}

patrimonio inmaterial

Figura 3: Mapa semántico: Patrimonio.

Fuente: A. Villalobos Gómez (2020)

Es así que las primeras actuaciones tuvieron lugar sobre el patrimonio arquitectónico y vinieron acompañadas de numerosas reflexiones sobre la definición del objeto patrimonial, los criterios de intervención o las necesidades de los usuarios, dando lugar a interesantes y decisivas teorías de la restauración formuladas por arquitectos, sean Viollet-le-Duc y la 'restauración estilística', Boito y la 'restauración filológica', Giovannoni y la 'restauración científica' o Annoni y la 'teoría del caso por caso'. Entre las cuestiones incorporadas por los arquitectos al debate patrimonial destacan el valor de uso entendido como un elemento que puede contribuir a la conservación de los bienes y la inserción de la huella contemporánea como un estrato irrenunciable en la historia del bien. Y tras la necesidad primera de consolidar las estructuras y conservar la materialidad de los bienes inmuebles, llegó más adelante la de ordenar y acondicionar su entorno, y por último la de exponer los bienes muebles asociados a esos edificios y lugares. De este modo se ha desarrollado una relación simbiótica entre arquitectura y patrimonio por medio del proyecto, que ha dado lugar a nuevas especialidades como la conservación del patrimonio, el paisajismo y la museografía.

Por patrimonio arquitectónico se entienden aquellos bienes culturales materiales que son de naturaleza inmueble y poseen un interés histórico, arqueológico, artístico, etnológico, científico, social o técnico. Desde el punto de vista espacial incluye elementos a diversas escalas, y desde el temporal elementos de distintas épocas, como resultado de un proceso histórico por el que de la idea inicial de 'patrimonio' (lo heredado) se llega a la de 'monumento' (lo digno de ser recordado), y actualmente 'bien cultural' (lo representativo de la civilización)

En definitiva, en el contexto actual el patrimonio ha pasado a entenderse como una construcción social por la que las personas se identifican con determinados bienes a los que conceden valor como monumento (valor estético), documento (valor científico), identidad (valor simbólico) y recurso (valor económico):

La idea de 'patrimonio' surge de un sentimiento de pérdida hace menos de doscientos años $y$, desde entonces, hemos recorrido un camino hacia el 'bien cultural' que nos ha llevado a un cambio de paradigma por medio de la desconstrucción del propio concepto, desligándolo de las connotaciones pasivas de pertenencia (lo heredado, objetual) a las de apropiación activa (lo generado, subjetual) (Villalobos Gómez, A 2017, p. 164).

Dichos bienes pueden ser de carácter material o inmaterial, si bien incluso los bienes inmateriales requieren para su manifestación del uso de determinados espacios, edificios u objetos que quedan asociados a través de dicho valor cultural. De este modo, el patrimonio arquitectónico también puede quedar vinculado a estos rituales, saberes y modos de expresión.

A su vez, el proyecto ha pasado a comprenderse como un acto cultural contemporáneo que establece una nueva relación con una arquitectura preexistente para explicitarlo:

El instrumento de intervención para la presentación y valorización de un sitio es el proyecto patrimonial [...] La supuesta objetividad de las decisiones técnico científicas debe ser sustituida por el acuerdo, tutelado por las administraciones responsables, de los técnicos y los gestores [...] El proyecto debe buscar el mejor conocimiento que podemos tener en e momento concreto que nos ha tocado vivir, aquello que ahora es racional creer pero que será sustituido en el futuro po otros parámetros más convenientes. Por ello, la intervención patrimonial debe aceptar que es en sí misma una 'construcción cultural’ (Fernández-Baca y Tejedor, 2007a, p. 78).

Por lo tanto, ni el patrimonio es algo dado que tenga valor exclusivamente por sí mismo ni el proyecto se puede entender solo desde las claves arquitectónicas, sino que debe integrarse en una estrategia patrimonia interdisciplinar. No tiene sentido hablar solo de 'patrimonio histórico' sino de 'patrimonio cultural' en su diversidad de clases y manifestaciones.

Patrimonio es el conjunto de las obras del hombre en las cuales una comunidad reconoce sus valores específicos y particulare y con los cuales se identifica. La identificación y la especificación del patrimonio es por tanto un proceso relacionado con la elección de valores. (Carta de Cracovia, 2000, anexo A).

El término 'valor' tiene su origen en la teoría de los valores planteada por el historiador Alois Riegl (1903) para analizar las razones para el 'culto moderno a los monumentos'. Confronta, por un lado, los valores 
que surgen del reconocimiento de la pertenencia de dichos bienes al pasado ('valores rememorativos') con la capacidad de los mismos para satisfacer, de manera similar a una obra recién creada, las necesidades materiales y espirituales de la sociedad ('valores de contemporaneidad'). A su vez, la teoría del 'bien cultural' enunciada por la Comisión Franceschini (1967) presenta el patrimonio como un elemento dual con un componente material dado por el soporte y una componente inmaterial como testimonio de la civilización. El término 'valor cultural' surge de la integración entre ambas teorías. Su carácter interdisciplinar viene dado por la diversidad de valores susceptibles de ser proyectados desde distintas disciplinas.

\subsection{Paisaje}

De la comprensión de la arquitectura como construcción del espacio existencial y del patrimonio como construcción cultural, deviene el paisaje como mirada intencionada, ya intuida por Le Corbusier cuando enunció que "La naturaleza se hace paisaje cuando el hombre la enmarca". La construcción no requiere una intervención física, sino que basta con la toma de conocimiento, es decir, la epistemología. El paisaje se ha instaurado, frente al monumento, como el nuevo paradigma de lo patrimonial: por sus cualidades materiales e inmateriales y su carácter holístico e integrador, configurado mediante procesos antrópicos sobre el medio natural, en el espacio y el tiempo (Figura 4). Se define como "cualquier parte del territorio tal como la percibe la población, cuyo carácter sea el resultado de la acción y la interacción de factores naturales y/o humanos" (Convenio Europeo de Paisaje, 2000, art.1).

\section{paisaje de interés cultural}

\section{paisaje natural paisaje cultulia paisajes intencionados, evolutivos y asociativos paisaje histórico urbano

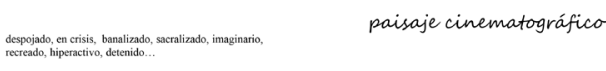 \\ paisaje agrario, costero, urbano $\cdots$}

Figura 4: Mapa semántico: Paisaje. Fuente: A. Villalobos Gómez (2020)

Si bien hasta 1972 no se formula el 'patrimonio natural' como una nueva categoría de protección en la Convención para la Protección del Patrimonio Cultural y Natural de UNESCO en París; hasta 1992 el Comité del Patrimonio Mundial no incorpora el 'paisaje cultural' como figura de protección, reconociendo tres categorías (Rössler, 2002): el 'intencionalmente creado por el hombre', el 'evolutivo' y el 'asociativo'. Se trata de un primer intento de definición con el que marcar los límites conceptuales.

El paisaje cultural es el resultado de la interacción en el tiempo de las personas y el medio natural, cuya expresión es un territorio percibido y valorado por sus cualidades culturales, producto de un proceso y soporte de la identidad de una comunidad (Plan Nacional de Paisaje Cultural, 2011, p. 22).
En verdad, todos los paisajes serían culturales, ya que no se trataría tanto de un lugar antropizado como de una mirada intencionada, al basarse su definición en las percepciones. "Todo paisaje, es decir, cualquier territorio, posee un cierto grado de naturalidad y de artificialidad, por ello, una posible categorización depende del grado de incidencia de uno u otro factor" (Menéndez de Luarca, 2006, p. 5).

La práctica ha venido a consolidar el uso del término 'paisaje' como concepto, ha reservado el 'paisaje cultural' para referirse a la figura de protección y ha incorporado el término 'paisaje de interés cultural' para precisar técnicamente aquellos paisajes significativos desde el punto de vista patrimonial, pero no declarados oficialmente (Plan Nacional de Paisaje Cultural, 2011, p. 28).

El Instituto Andaluz del Patrimonio Histórico (en adelante, IAPH) establece seis grandes sistemas de referencia patrimonial (Rodrigo et al., 2012, p. 70): de asentamientos, de infraestructuras territoriales, de seguridad y defensa, de producción y transformación, de creencias, artes y comunicación, y una última categoría referida a accidentes geográficos connotados. Se trata de una propuesta metodológica de enorme validez para la elaboración de registros de paisajes. En otros niveles, incorporan denominaciones más descriptivas que se refieren al soporte territorial: agrario, costero, urbano, etc. Por último, existen denominaciones más sugerentes, como la del arquitecto Pedro Salmerón (2003), de carácter valorativo: despojado, en crisis, banalizado, sacralizado, imaginario, recreado, hiperactivo o detenido. De nuevo el paisaje se diversifica en terrestre-celeste, histórico-contemporáneo, vivo-fósil, etc.

El paisaje no es un ente objetual ni un conjunto de elementos físicos cuantificables, tal como lo interpreta las ciencias positivas, sino que se trata de una relación subjetiva entre el hombre y el medio en el que vive, relación que se establece a través de la mirada (Maderuelo, 2005, p. 12).

En un campo de conocimiento donde cada vez confluyen más disciplinas, la normalización terminológica resulta fundamental para entenderse y apreciar matices. Las dificultades de gestión del paisaje cultural se derivan de su propia vulnerabilidad y de la diversidad de escalas, enfoques, disciplinas y agentes.

\section{Resultados}

Investigar en Patrimonio (Figura 5) significa producir un conocimiento innovador relativo a los bienes culturales que redunde en su conservación y disfrute. Se trata de una de las acciones de tutela patrimonial, junto a la documentación, protección, conservación, presentación y difusión, necesaria para garantizar no solo la salvaguarda de la materialidad de los bienes, sino especialmente sus valores culturales.

Investigar en Arquitectura significa producir un conocimiento innovador relativo al espacio existencial del hombre. Dado el doble carácter humanista y técnico de la disciplina, el tipo de conocimiento producido puede ser analítico (creación basada en la ciencia), sintético (aplicación basada en la ingeniería) o simbólico (resignificación basada en el arte): por lo 
tanto, la innovación puede consistir tanto en una idea como en un objeto arquitectónico. Cuando la temática arquitectónica ha sido abordada desde metodologías tradicionales (filosófica, histórica, empírica) no ha sido necesario justificar la componente investigadora. En cambio, sí se ha demandado explicitarla al hacer uso de instrumento específico de la arquitectura: el 'proyecto'. Se entiende que esta dificultad haya radicado en su marcado enfoque propositivo, obviando que el proyecto no es solo el fin sino el medio para alcanzar el resultado. De este modo, en el proyecto confluyen los objetivos de investigación e intervención en una misma herramienta procedimental y operativa. Esto explica que sea habitual afrontar la investigación en arquitectura, más que como investigación básica, como investigación aplicada y/o desarrollo tecnológico; $y$, a nuestro modo de ver, que se derive que en arquitectura no solo hay que investigar para intervenir sino también intervenir para investigar.

\section{conocer para intervenir \\ investigación aplicada interdis c1p prórecteriedac divulgar planificar identificar diagnosticar G Vallorar}

Figura 5: Mapa semántico: Investigar

Fuente: A. Villalobos Gómez (2020)

Referido al patrimonio arquitectónico, podemos entender que investigar consiste en las siguientes acciones de carácter propositivo, de menor a mayor escala:

\subsection{Realizar el registro gráfico de un bien cultural}

La documentación gráfica de un bien cultural es el primer paso para su conocimiento y protección, a través de la fotografía como instrumento de representación de una realidad inmediata, y del dibujo como instrumento de análisis complementario.

Un ejemplo paradigmático de investigación arquitectónica a partir del análisis de los registros gráficos sería e llevado a cabo en el Conjunto Arqueológico Dólmenes de Antequera. EI CADA es la institución museística de la Junta de Andalucía que, desde 1986, tiene encomendada la tutela y valorización del Bien de Interés Cultural, con la tipología de Zona Arqueológica, denominado 'Dólmenes de Antequera' y que, desde 2016, está encargada de representar y hacer un seguimiento de la gestión del bien Patrimonio Mundial denominado 'Sitio de los dólmenes de Antequera'. Esta institución realizó una búsqueda de documentación gráfica para la definición de su repertorio gráfico que se remontó a una primera planimetría de dolmen de Menga, realizada en 1847 por el arquitecto Rafael Mitjana (Ruiz González, 2011, tomo I, p. 186). Estos planos le darían difusión internacional (incorporando Menga en las discusiones científicas) y en adelante fijarían una iconografía (reproducida e interpretada por otros investigadores). Del repertorio gráfico encontrado, llama la atención por su originalidad un dibujo realizado en 1853 por la viajera Louisa Tenison desde el corredor hacia el exterior (Ruiz González, 2011, tomo I, p. 187).

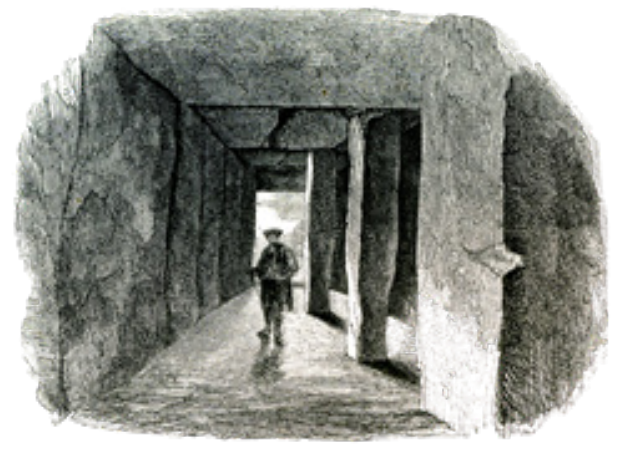

Figura 6: Interior of the cave. Louisa Tenison, 1853 Fuente: Conjunto Arqueológico Dólmenes de Antequera

Entre otras cuestiones, esta autora intuye la inusual orientación del dolmen hacia la Peña, a pesar de la presencia intermedia de un olivo centenario. En 2005 con la retirada del olivo, el fotógrafo Javier Pérez González verificaría gráficamente por primera vez dicha alineación (Villalobos Gómez, 2020a, p. 36) y sería el arqueoastrónomo Michael Hoskin quien confirmara la excepcionalidad de esta orientación terrestre, dando cumplimiento al criterio iii para la declaración del Sitio de los Dólmenes de Antequera como Patrimonio Mundial (UNESCO, 2016, p. 224).

Disponer de un registro gráfico actualizado de los bienes culturales es fundamental para su tutela, no solo a nivel de documentación sino a efectos de su protección y difusión. En este sentido los SIG (Sistemas de Información Geográfica) y los BIM (Building Modelling Information) se han incorporado para completar el rigor de la información y ampliar sus posibilidades al poder ubicar los bienes espacialmente, visualizar de manera inmediata en los planos los datos asociados en sus tablas de atributos y, sobre todo, evitar la dispersión de información gráfica y/o textual emitida por distintas fuentes. En el año 2005, el Conjunto Arqueológico de Carmona desarrolló un sistema de información denominado SICAC, convirtiéndose en el primer yacimiento con una réplica virtual de todas sus estructuras arqueológicas a efectos de su documentación y disponible online para una mejor difusión de los bienes (Jiménez, López y Rodríguez, 2010). Igualmente, el Conjunto Arqueológico de Itálica planteó en el año 2009 la gestión de la información gráfica desde un sistema AMEIM (Archaelogical and Monumental Ensembles Information Modeling) que combinara las prestaciones SIG y BIM al interrelacionar el modelo gráfico y la base de datos para la consulta y análisis dirigido a la toma de decisiones de conservación (Rodríguez de Guzmán, 2011, p. 146).

\subsection{Analizar el proceso creativo de un proyecto}

No necesariamente tiene que tratarse de un proyecto arquitectónico en el sentido estricto sino de la instalación, permanente o efímera, de una obra artística cuando genera un lugar en un entorno patrimonial. 
Sirva como ejemplo la brillante intervención Dove l'arte ricostruisce il tempo, planteada en 2016 por el artista Edoardo Tresoldi, consistente en la reconstrucción volumétrica con redes metálicas de la basílica paleocristiana de Santa María la Mayor, en el marco de Proyecto de restauración y recualificación del Parque Arqueológico de Siponto dirigido por el arquitecto Francesco Longobardi en Manfredonia, Italia (Venere, 2016). Se trata de una intervención aparentemente sencilla que cumple con los criterios consensuados en patrimonio cultural (discernibilidad, reversibilidad, mínima intervención y compatibilidad de materiales). Al mismo tiempo ofrece una imagen absolutamente contemporánea en la que también cabe el paso de tiempo (la oxidación del metal, el crecimiento de la maleza o el anidamiento de aves). La intervención contemporánea dota de singularidad una preexistencia que, de otro, modo, permanecía muda en su contexto original.

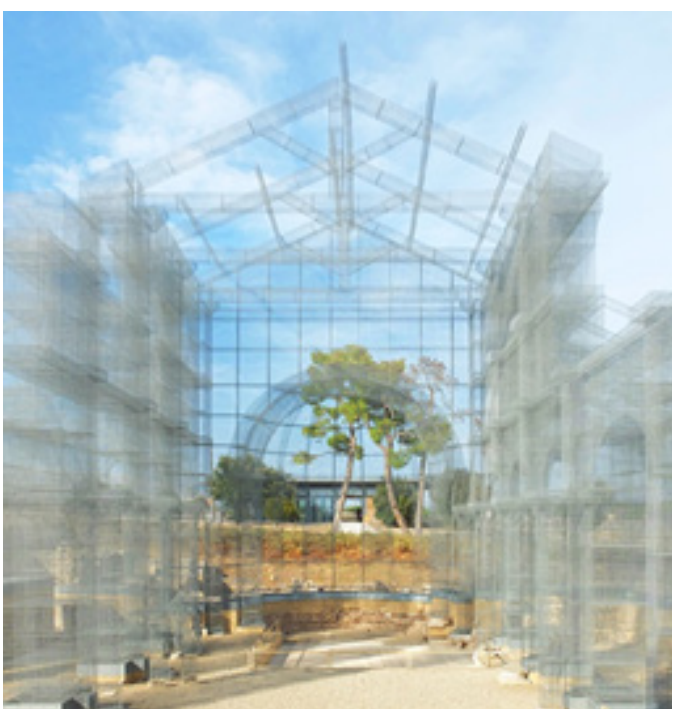

Figura 7: Intervención artística en la Basílica de Siponto, Manfredonia (Italia). Edoardo Tresoldi, 2016 Fuente: Fabio Omero (2017). CC BY-NC-SA 2.0

\subsection{Reconocer los valores patrimoniales de un bien para definir la estrategia de protección}

La investigación, entendida como producción y transferencia de conocimiento, es imprescindible para la toma de decisiones razonadas, transparentes y eficaces. Es la acción fundamental para la tutela de los bienes culturales, ya que descubre los aspectos singulares de dicho bien y lo pone en contexto con otros.

Resulta crucial para fundamentar el valor cultural de los patrimonios emergentes, sobre todo el patrimonio arquitectónico contemporáneo, por su proximidad temporal. En el año 2012 fue inscrita la ciudad de Río de Janeiro como Patrimonio Mundial en la categoría de paisaje cultural, a pesar de no tratarse de un jardín histórico o un sistema agrícola tradicional (UNESCO, 2012).
También es importante revisar los valores culturales de un bien a la luz de nuevas investigaciones. Sea el caso del Santuario de Nuestra Señora de Cabo Espichel o de Pedra da Mua, en Sesimbra, Portugal. Se trata de un lugar de peregrinación, emplazado próximo a un acantilado en el Parque Natural de la Sierra de Arrábida. Su origen se remonta al siglo $\mathrm{XV}$, si bien el conjunto arquitectónico data de los siglos XVII-XVIII. La tradición cuenta que la Virgen se apareció a dos vecinos en la cima de dichos acantilados montando una gigantesca mula y que sus huellas quedaron marcadas en la piedra, tal y como se representa en uno de los azulejos del edificio denominado la Ermita de la Memoria. En 1971 se estudiaron unas marcas en dichos acantilados y se identificaron con huellas de desplazamiento dejadas por una manada de dinosaurios saurópodos, a finales del Jurásico superior. Constituyen el primer ejemplo contrastado de comportamiento gregario en saurópodos reconocido en un yacimiento europeo; y el azulejo que relata la leyenda constituye la primera figuración de icnitas de dinosaurio del mundo (Alcalá et al., 2018, p. 118). De este modo, lo que no hubiera dejado de ser una iglesia barroca más en un sitio pintoresco, con todo su valor histórico, artístico, devocional, antropológico y paisajístico, adquiere un inimaginable valor paleontológico de carácter excepcional, rescatado a partir del trabajo de campo, la investigación documental y el análisis iconográfico de sus representaciones artísticas.

De esta manera, el patrimonio natural queda vinculado al cultural no solo a través de sus elementos materiales sino de un patrimonio inmaterial que reinterpreta los signos del paisaje en una clave trascendente, por medio de la tradición oral o las manifestaciones artísticas. Sean las huellas de dinosaurio de La Rioja que, por su proximidad al Camino de Santiago, pasan por ser las huellas del caballo del apóstol o la elección de unas rocas con abundantes icnitas para excavar unas tumbas en las necrópolis medievales de Regumiel de la Sierra y Revenga, en Burgos (IGME)

\subsection{Identificar un paisaje cultural para esta- blecer líneas de intervención}

EI IAPH lleva desarrollando desde hace varios años una línea de investigación pionera sobre paisaje. Dado que los paisajes están conformados por bienes heterogéneos en los que se da una dialéctica continua de permanencia y cambio debido al uso, era necesario proponer una metodología para su identificación y protección. En el año 2004 redactó la Guía del paisaje cultural de la Ensenada de Bolonia (Salmerón, 2004), un documento de análisis previo que caracterizó este ámbito patrimonial a través del lugar, las acciones y las percepciones, estableció los criterios de actuación para su conservación y definió acciones y transformaciones para: fomentar los recursos culturales, proteger y mejorar el medio físico, modernizar las infraestructuras, planificar el territorio y adecuar las actividades económicas a los valores reconocidos. En la identificación de dicho paisaje, con una importante componente natural, lo más difícil fue delimitar el ámbito de estudio e incorporar en las propuestas su carácter antropizado. 
En el año 2015 se traslada esta metodología de reconocimiento de la estructura del paisaje a un contexto urbano con la Guía del Paisaje Histórico Urbano de Sevilla (Fernández-Baca, Fernández y Salmerón, 2015). En este caso, las cuestiones más complejas de abordar fueron la inserción de la arquitectura contemporánea en los centros históricos y la valoración patrimonial de las periferias urbanas. En patrimonio cultural, la comprensión de la vida del bien desde la lógica histórica conlleva el reconocimiento de todas las épocas como presentes pretéritos que aspiran a dejar su huella. Sin embargo, se sigue produciendo una distancia mental hacia e patrimonio contemporáneo por la que, en todo caso, es más fácil entender un edificio de nueva planta (como monumento) que una intervención contemporánea en un contexto patrimonial (como documento). Pensamos que esto suceda porque la conservación se malinterpreta en oposición al uso, cuando lo cierto es que los productos culturales de la modernidad no solo pueden convertirse en bienes a proteger, sino que además nos pueden ayudar a explicitar o trascender los valores propios de las preexistencias. Esta incomprensión social se manifiesta con la oposición casi sistemática a cualquier intervención sobre el patrimonio consolidado. Tenemos por delante una importante tarea de difusión para que se resuelva e debate sobre la inserción de la arquitectura de nuestro tiempo; superado por los especialistas Cesare Brandi y Roberto Pane a finales de los años cincuenta (Gurrieri, 1977, pp. 55-78); porque de otro modo, si se insiste en poner un límite artificial a lo contemporáneo, no solo perderemos la mejor huella de nuestro tiempo, sino que probablemente perdamos la oportunidad de singularizar un patrimonio heredado común.

\subsection{Estudiar la viabilidad de uso de un edifi- cio con valores patrimoniales para proponer un programa de usos compatible}

Las cartas internacionales coinciden en que el uso es un factor que garantiza la conservación de un monumento cuando es compatible con su tipología arquitectónica, respeta sus valores culturales y se dedica a una función útil a la sociedad. La ciudad histórica posee determinados contenedores vacíos que, por su tipología, no pueden volver a tener su uso primigenio, pero están a la espera de otro compatible que permita su rehabilitación. Es asi que la ciudad de Sevilla cuenta con unas atarazanas del s. XIII, que hasta finales del s. XV fueron el mayor complejo industrial del principal puerto de Europa. Dada su situación estratégica y su versatilidad espacial, el edificio fue diversificando sus usos y se fragmentó hasta llegar abandonado al siglo XXI. Desde la consideración de que la estrategia de la visita cultural no puede ser la única alternativa de reúso, el IAPH realiza, en el año 2007, un análisis técnico de sus valores patrimoniales y del contexto cultural en el que se inserta para concluir una propuesta de uso coherente que permita recuperarlo para la ciudad como un generador de usos (FernándezBaca, 2007b). Se trata de una investigación impecable y necesaria para una gestión eficiente de nuestro patrimonio construido, histórico o contemporáneo.

Otra cuestión es que el proyecto no se haya ejecutado todavía por divergencias en los criterios de intervención, que llegaron hasta los tribunales y que han condicionado erróneamente la comprensión del monumento a su momento fundacional. El debate no se ha centrado tanto en conservar/restaurar como en reivindicar/ desactivar un proyecto arqueológico que, en nombre de la autenticidad del monumento, altera las condiciones del terreno, el equilibrio de cargas de la estructura y la espacialidad interior. De ser finalmente así, resultará difícil la valorización de un 'espacio arqueológico' que se plantea como una discontinuidad espacial, constructiva y cronológica del resto del edificio y que además ha quedado descontextualizado de un paisaje urbano que también se ha visto transformado por la propia lógica de los sedimentos fluviales y las dinámicas sociales.

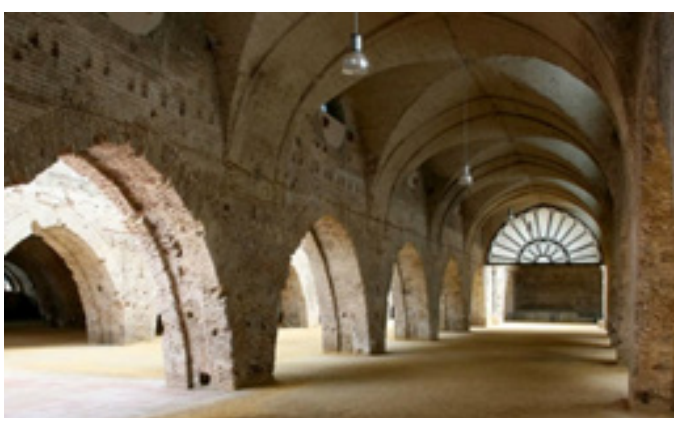

Figura 8: Atarazanas, Sevilla (España)

Fuente: A. Villalobos Gómez (2006)

Por supuesto, esta acción investigadora abre una línea muy interesante sobre la gran capacidad de transformación de los edificios históricos como contenedores multifuncionales a pesar de estar diseñados con tipologías, trazas y esquemas muy ajustados a su función primigenia.

4.6. Diagnosticar el estado de conservación de un bien inmueble o mueble para proponer posibles criterios de intervención y pautas de mantenimiento

Antes de intervenir un bien hay que conocer su estado de conservación y los valores culturales a proteger para poder avanzar criterios y actuaciones. En el caso de patrimonio arquitectónico nuestro diagnóstico suele centrarse en un edificio o lugar, pero se pueden dar otras situaciones con determinados bienes muebles (pinturas murales, retablos y artesonados), que sería preciso abordar desde la arquitectura debido a sus dimensiones, comportamiento estructural o medios técnicos para la intervención.

4.7. Estudiar el impacto patrimonial de una actuación que pueda incidir en el entorno de un bien cultural

A diversas escalas, las administraciones responsables de la tutela del patrimonio deben prever las posibles incidencias de otras actuaciones, culturales o no, sobre los valores de dichos bienes protegidos. Es así que de manera sistemática se realizan seguimientos o estudios arqueológicos: con carácter previo a la excavación para 
la cimentación de un edificio de nueva planta en un conjunto histórico, un movimiento de tierras en una zona de servidumbre arqueológica, o de urgencia, con ocasión de hallazgos casuales en el trazado de una infraestructura viaria.

Esta cuestión la tiene bien presente la UNESCO cuando, en la candidatura de un bien para Patrimonio Mundial, solicita estudiar el impacto patrimonial de otras actuaciones en el área protegida o zona de amortiguamiento. Durante el proceso de candidatura del Sitio de los Dólmenes de Antequera a Patrimonio Mundial se tuvo que resolver el impacto de un edificio preexistente sobredimensionado en el área arqueológica y el desarrollo urbanístico de un polígono industrial para reducir la contaminación visual. Esta experiencia de planificación ha servido como modelo para incorporar en el Anteproyecto de la Ley de Patrimonio Histórico de Andalucía el Informe de Valoración de Impacto Patrimonial como un documento obligatorio, impuesto al titular de cualquier proyecto que incida en elementos de Patrimonio Mundial situados en Andalucía, con objeto de identificar los potenciales impactos y definir las medidas de mitigación o de compensación que aseguren que e Valor Universal Excepcional del elemento integrante del Patrimonio Mundial afectado no se vea impactado negativamente (Anteproyecto LPHA, 2018, pp. 4-5).

4.8. Intervenir en un edificio o un lugar con valores patrimoniales por motivos de conservación, adecuación funcional y/o valorización para el uso y disfrute de la sociedad

Todo proyecto de intervención tiene una componente investigadora irrenunciable ya que a los problemas planteados por el bien le siguen las soluciones planteadas de manera coherente y razonada en una propuesta.

El primer proyecto conocido de musealización in situ en un yacimiento arqueológico es la cubrición de la Villa del Casale en Piazza Armerina (Sicilia), por el arquitecto Franco Minissi, en 1957. Se trataba de cubrir la mayor colección de mosaicos romanos del mundo, descubierta solo unos años antes. La cuestión de la musealización in situ de los restos arqueológicos aún era incipiente en aquella época, pero la acomete confiado en las posibilidades de la arquitectura contemporánea y bajo la supervisión del historiador Cesare Brandi (Alagna, 2008, p. 40). Para ello plantea una novedosa estructura de pasarelas (que permiten la contemplación de los mosaicos sin desgastarlos por el uso) y una sutil cubierta transparente (no unitaria ni mimética, que recupera la escala de los espacios de la villa sin arrojar sombras a pavimento) y ventilada (mediante una cámara de aire intermedia que mantiene el equilibrio higrotérmico).

La propuesta resolvía de manera impecable la imagen general del yacimiento, garantizaba la conservación preventiva de los bienes muebles y mejoraba las condiciones de visita, desde un punto de vista teórico: motivada por la conservación, discernible y reversible. El arquitecto comprende el proyecto como un acto crítico y la museografía como una actuación de conservación preventiva. El proyecto de Minissi no solo refleja un estrato moderno en la vida concreta del bien cultural,

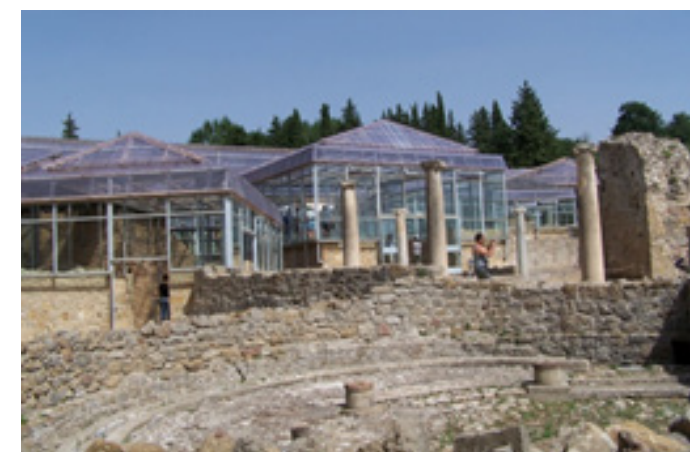

Figura 9: Villa del Casale, Piazza Armerina (Italia). Franco Minissi, 1957

Fuente: Peppe64 (2007). CC BY-SA 3.0

sino un modo de intervención en la historia de la teoría de la restauración, por lo que esta situación nos plantea hasta dónde debe llegar la revisión de su obra como parte de la preexistencia. Ya no se trata solo de la villa romana de finales de siglo III d.C sino de un bien arqueológico intervenido en 1957, declarado Patrimonio Mundial en 1997.

En el caso límite de edificios deteriorados en procesos traumáticos, como la Catedral de Notre-Dame de París tras el incendio de su cubierta, la investigación patrimonial será imprescindible para el discernimiento de los criterios de intervención.

\subsection{Participar en la redacción de un plan direc- tor para planificar la tutela de un bien cultural}

Un plan es una herramienta de planificación que ordena objetivos y actuaciones, estableciendo una secuencia de prioridades. En Andalucía, el Plan Director es la figura de gestión, que ordena las actuaciones de tutela de sus espacios culturales desde el año 2007. Tiene su precedente en la intervención del Monasterio de la Cartuja de Santa María de las Cuevas en Sevilla (19891992), bajo la coordinación del Arqueólogo-Conservador del Patrimonio Bartolomé Ruiz. Se redacta un documento de planificación que se despliega en cinco proyectos de arquitectura con un amplio equipo interdisciplinar. Tuvo por objeto la rehabilitación del conjunto monumental como sede del Pabellón Real para la Exposición Universal de 1992, previendo la posterior transformación del conjunto como sede de tres instituciones culturales que al día de hoy se mantienen: el Instituto Andaluz de Patrimonio Histórico, el Centro Andaluz de Arte Contemporáneo y la Universidad Internacional de Andalucía. Obtuvo el Premio Nacional de Restauración de Monumentos, concedido por la Real Fundación de Toledo en 1993.

El primer Plan Director redactado por un conjunto arqueológico en España conforme a los Criterios para la elaboración del Plan Museológico fue el CADA, en el año 2011, con su Avance del Plan Director 2011-2018. Supuso un documento novedoso por la manera de resolver la dificultad de adaptar un documento técnico pensado para un edificio. Posteriormente se ha redactado su Plan Director 2018-2025, conforme a la Orden de 3 de marzo de 2016, por la que se aprueba las directrices técnicas 


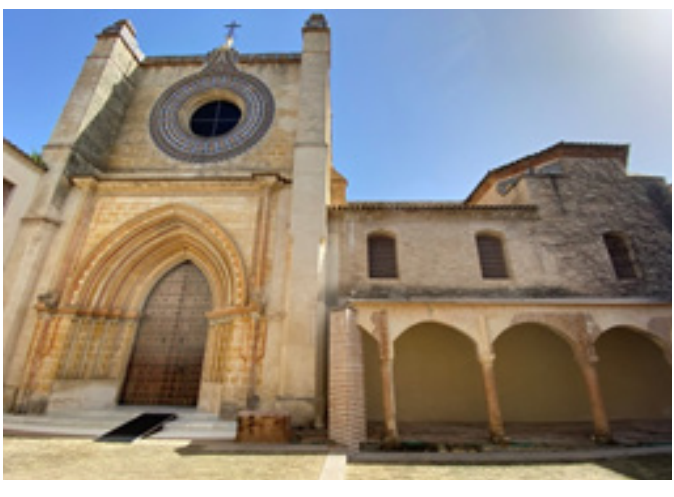

Figura 10: Cartuja de Santa María de las Cuevas, Sevilla (España). José Ramón Sierra, 1992

Fuente: A. Villalobos Gómez (2021)

para la elaboración de los documentos de planificación y evaluación de los museos, colecciones museográficas y conjuntos culturales de Andalucía (BOJA no56, de 23 de marzo de 2016), siendo la primera institución museística de Andalucía en adoptarlo. Ha supuesto un proceso de reflexión sobre las particularidades en la tutela de los conjuntos arqueológicos en cada uno de sus programas.

\subsection{Participar en la redacción de proyectos museológicos y museográficos para explicitar el valor cultural}

Una de las posibles aplicaciones de la investigación patrimonial consiste en la redacción de un discurso museológico y el diseño de la museografía de una exposición, considerando los valores del edificio y sus colecciones, los requerimientos de los bienes expuestos y las prestaciones de los visitantes para optimizar la relación entre continente y contenido.

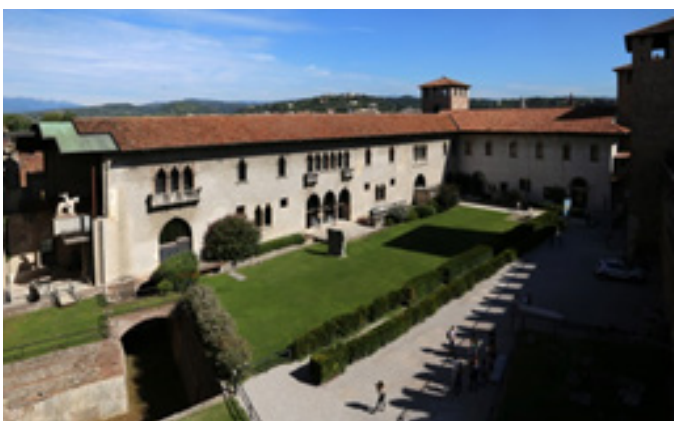

Figura 11: Museo de Castelvecchio, Verona (Italia). Carlo Scarpa, 1958-1975

Fuente: Sailko (2019). CC-BY-SA-4.0

Un primer ejemplo es la intervención en el Museo de Castelvecchio en Verona, de Carlo Scarpa, entre 1958 y 1975, como proyecto museográfico ejecutado. Supone una referencia obligada en lo que se refiere a la musealización del propio edificio para contar su historia y presentar sus colecciones desde un lenguaje contemporáneo (Lieto, 2006). El proyecto no distingue entre contenedor y contenido, sino que de la relación entre ambos surge el discurso museológico. La intervención se reconoce por la presentación de la escultura ecuestre de Cangrande en un punto crucial del recorrido, donde el muro permite leer la historia de edificio por medio de su secuencia estratigráfica, y la cubierta se descompone constructivamente en un gesto poético de protección. El acierto de la intervención se evidencia cuando sirve de inspiración tanto a proyectos de conservación del patrimonio como de obra nueva; no cabe en esta preguntarse dónde está el límite entre el palacio medieval y el museo contemporáneo, dónde queda el arquitecto conservador que hace pedagogía del edificio y el arquitecto moderno que diseña soportes accesibles a la vez que escaleras imposibles. En definitiva, se trata de una propuesta integral que cubre, de manera magistral y a diversas escalas, las necesidades (a veces incompatibles) de los bienes y los usuarios.

Un segundo ejemplo es el Proyecto Museológico del Sitio de los Dólmenes de Antequera (Villalobos Gómez, 2021), como documento técnico ejecutable que define y desarrolla los contenidos expositivos de este bien Patrimonio Mundial y su museo de sitio. Se estructura en dos bloques: el proyecto museológico de la zona arqueológica que define las áreas temáticas para la presentación in situ de los bienes inmuebles y el proyecto museológico del museo de sitio, que define las áreas temáticas de la exposición permanente 'Antequera Milenaria' para la presentación de los bienes muebles asociados. El hilo conductor es el fenómeno de monumentalización paisajística que se da entre los tres bienes culturales (dólmenes de Menga y Viera y tholos de El Romeral), y los dos bienes naturales (La Peña de los Enamorados y la sierra de El Torcal). En ambos casos los contenidos se apoyan en las evidencias materiales que suponen la presencia auténtica e íntegra de los tres megalitos y los restos arqueológicos hallados en el territorio de las comunidades que los construyeron. Se trata de un documento innovador en la medida en que, por primera vez, no limita las áreas temáticas a la exposición permanente del museo de sitio (discurso de la colección), sino que incorpora el propio yacimiento a modo de un museo en el territorio (discurso del paisaje) que dota de sentido al anterior. La arquitectura prehistórica recupera su conexión con el paisaje y la arquitectura contemporánea del museo de sitio recoge las mismas claves paisajísticas en su implantación.

\subsection{Diseñar un itinerario cultural consideran- do las posibles redes y nudos patrimoniales}

Otra intervención paradigmática en patrimonio es la que diseñó entre 1954 y 1958 el arquitecto Dimitris Pikionis en la colina de Filopapo en Atenas, para acceder a la Acrópolis desde una colina contigua. Se trata de un camino de 520 metros ejecutado con materiales cerámicos, cornisas y restos de mármoles procedentes del derribo de casas en el siglo XIX y franjas de hormigón ejecutadas in situ que se adaptan a las discontinuidades del terreno, tales como piedras emergentes y troncos de árboles. Esta intervención urbana eleva los escombros a la categoría de ruinas contemporáneas que dialogan con la memoria del paisaje (García, 2015).

Otro tema de interés para la investigación arquitectónica es el trazado de un sendero efímero como el proyecto Floating Piers. 


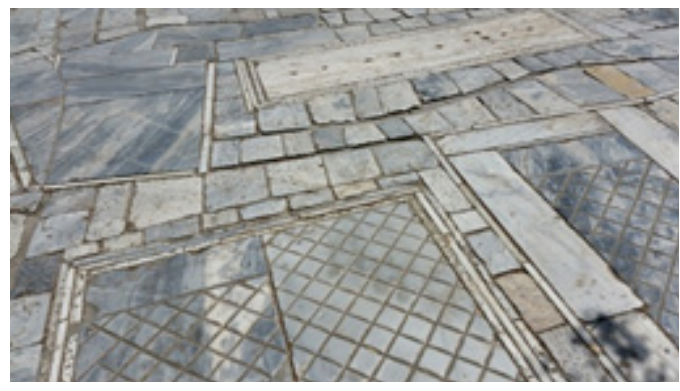

Figura 12: Camino de Filopapo, Atenas (Grecia). Dimitris Pikionis, 1954-1958

Fuente: Marcela Cernadas (2015)

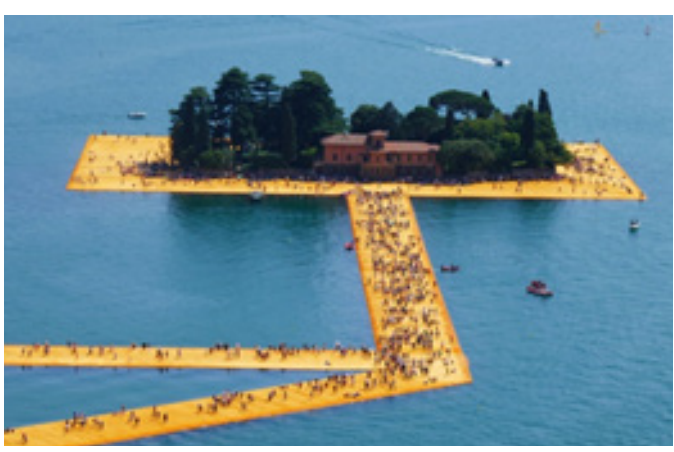

Figura 13: Floating Piers, Lago Iseo (Italia). Christo y Jeanne Claude, 2016

Fuente: Newton Court (2016). CC-BY-SA-4.0

Los artistas Christo y Jeanne Claude construyen en el año 2016 una pasarela flotante de tres kilómetros de longitud y 16 metros de anchura sobre el Lago Iseo para unir, durante dieciséis días del verano, las calles peatonales de la ciudad de Sulzano con las islas de San Paolo y Monte Isola. Son muchas las cuestiones de interés: los aspectos técnicos (sistema constructivo, condiciones de estabilidad, características de los materiales), los aspectos administrativos (estrategia de gestión para su viabilidad, solicitud de permisos, coordinación de los municipios), y especialmente los aspectos patrimoniales (impacto patrimonial, enfoque paisajístico, repercusión en el imaginario cultural y actitudes de la población local).

\section{Conclusiones}

Respecto a la deriva conceptual, la investigación nos ha permitido discernir los conceptos fundamentales de los que emanan los demás, explicitando además el factor común existente entre el espacio, la arquitectura, el patrimonio y el paisaje. De esta manera el espacio arquitectónico resulta ser la concretización de un espacio existencial o lugar reconocible por quien despliega su identidad en él; la arquitectura se comprende como un trozo de aire humanizado que no tiene que corresponderse estrictamente con una construcción física, sino con una manera de habitar o cuidar el espacio; el patrimonio arquitectónico es una construcción social basada en una valoración de tipo cultural sobre la arquitectura; y el paisaje supera la idea de espacio natural que actúa como mero contexto físico que condiciona las premisas del proyecto arquitectónico para convertirse en una mirada epistemológica. Por lo tanto, esa concretización, modo de habitar, valoración cultural o mirada epistemológica, están relacionadas porque, en definitiva, son una manifestación de la relación entre las personas y su medio.

Respecto a la forma de generación de conocimiento sobre el patrimonio desde la arquitectura, se han identificado once acciones planteadas desde esta idea amplia de arquitectura (como registro gráfico, hecho artístico, investigación espacial, explicitación de valores, soluciones funcionales) con incidencia en el patrimonio cultural en sus diversas escalas (la del bien mueble, el monumento, la ciudad patrimonial histórica o contemporánea y el territorio). Esta epistemología del paisaje arquitectónico nos muestra que las posibilidades de investigación en el patrimonio arquitectónico no se limitan al estudio de la historia de un edificio o de su estado de conservación, sino a todas las cuestiones abordables desde las capacidades de creación y planificación de la propia disciplina arquitectónica. Siendo cada bien único, también lo serán las propuestas planteadas en ellos, pero este enfoque permite abrir el camino a cierta normalización de la generación del conocimiento en función de la finalidad y escala de actuación.

Respecto al estudio de casos planteados para cada línea de acción, se detecta que la mayoría de las propuestas seleccionadas abarcan el ámbito mediterráneo, en un marco temporal que comprende desde 1853 a 2016, sobre edificios de distinta tipología (residenciales, civiles, religiosos), cronología (prehistórico, clásico, medieval, moderno y contemporáneo) y paisajes (urbano, rural y marítimo). Por supuesto, un ejercicio teórico-práctico imprescindible sería profundizar en cada uno de los ejemplos para reconocer nuevas referencias conceptuales (un mapa de proyectos patrimoniales relacionados) y estudiar el proceso creador (de la idea a la realidad). En relación con las nuevas referencias conceptuales, sería muy interesante contrastar con ejemplos de otro ámbito cultural para analizar las analogías y divergencias en las formas de generación de conocimiento patrimonial: comparando cronologías, tipologías y paisajes. En relación con el proceso creador, otra posible línea de investigación sería valorar cómo dichas intervenciones no han sido ideadas y/o ejecutadas exclusivamente por arquitectos, lo que no le resta carácter arquitectónico a la intervención. En muchos casos se han planteado como intervenciones artísticas que, partiendo de formatos como la escultura o el land art, han requerido posteriormente la participación de arquitectos para la construcción de la idea. Las posibilidades de análisis no se agotan tampoco en la disciplina arquitectónica, sino que resulta absolutamente pertinente y enriquecedor abordar complementariamente estas cuestiones desde la teoría de las representaciones sociales (del psicólogo social Serge Moscovici) o del imaginario colectivo (del filósofo y sociólogo Edgar Morin), al tener como punto de partida el patrimonio como construcción social en la que un conjunto de individuos reconocen representaciones, valores y prácticas culturales comunes.

En definitiva, las posibilidades de generación de conocimiento patrimonial desde la arquitectura 
son innumerables porque 'investigar' es una acción fundamental en la construcción social del 'patrimonio', y la 'arquitectura' nunca dejará de 'crear' y 'planificar' nuevos espacios existenciales.

Cómo citar este artículo/How to cite this article: Villalobos Gómez, A (2021). Epistemología del paisaje arquitectónico. La generación de conocimiento patrimonial desde la arquitectura. Estoa. Revista de la Facultad de Arquitectura y Urbanismo de la Universidad de Cuenca, 10(20), 103-115. doi: https://10.18537/est.v010.n020.a09

\section{Referencias bibliográficas}

Alagna, A. (2008). Franco Minissi. Restauro e musealizzazione dei siti archeologici in Sicilia. Università degli Studi di Napoli Federico II.

Alcalá, L., Cobos, A. y Royo-Torres, R. (2018). Dinosaurios de la Península Ibérica. Revista PH, (94), 116-153.

Bonaiti, M. (Selec.)(2002). Architettura è Louis I. Kahn, gli scritti. Electa.

Christo y Claude. J. (2016). The Floating Piers. https:// christojeanneclaude. net/projects/the-floating-piers

Conferencia Internacional sobre Conservación (2000). Carta de Cracovia. Principios para la conservación y restauración del patrimonio construido.

Ferlenga, A. (1999). Dimitris Pikionis 1887-1968. Electa.

Fernández-Baca Casares, R., Fernández Cacho, S. Y Salmerón Escobar, P. (Coord.) (2015). Guía del Paisaje Histórico Urbano de Sevilla. IAPH.

Fernández-Baca Casares, R. (Dir.) (2007b). Estudio de viabilidad y adecuación como generador de usos para las Reales Atarazanas de Sevilla. IAPH [informe inédito].

Fernández-Baca Casares, R. y Tejedor Cabrera, A. (2007a). Conservación y uso de los teatros romanos: el caso del teatro romano de Málaga. Mainake, (29), 77-101.

Ferrater Mora, J. (1965). Diccionario de Filosofía. [1a ed. 1941]. Editorial Sudamericana.

García Sánchez, J.F. (2015). Dimitris Pikionis: la alfombra pétrea. Revista PH, (87), 232-233.

González-Calero, A. (1998). Miguel Fisac: la arquitectura es un trozo de aire humanizado. Añil, Cuadernos de Castilla-La Mancha, 3 (14), 42-46.

Gurrieri, F. [Selec.] (1977). Teoria e cultura del restauro dei monumento e dei centri antichi. Cooperativa Editrice Universitaria.

Heidegger, M. (1994). Conferencias y artículos. [1 1 ed. 1951]. Ed. del Serbal.

Instituto del Patrimonio Cultural de España (2011). Plan Nacional de Paisaje Cultural. IPCE.

Jiménez Hernández, A., López Sánchez, J.M y Rodríguez Temiño, I. (2010). Sistema de información del Conjunto Arqueológico de Carmona. Sevilla. España. Virtual Archaeology Review, 1 (2), 117-122.

Junta de Andalucía (2018). Anteproyecto de Ley por el que se modifica la Ley 14/2007, de 26 de noviembre, de Patrimonio Histórico de Andalucía.

https://www.juntadeandalucia.es/servicios/normaselaboracion/detalle/138452.html 
Lalande, A. (1966). Vocabulario técnico y crítico de la Filosofía. Editorial El Ateneo.

Lieto, A. (2006). I disegni di Carlo Scarpa per Castelvecchio. Marsilio.

Maderuelo, J. (Dir.) (2010). Paisaje y patrimonio. Abada Editores.

Menéndez de Luarca Navia-Osorio, J. R. (2006). La arqueología del paisaje y el paisaje de la arqueología: el plan especial de protección de Madinat al Zahra. https://joseramonmenendezdeluarca.files.wordpress. com/2015/04/la-arqueologc3ada-del-paisaje-y-elpaisaje-de-la-arqueologc3ada.pdf

Navarro Baldeweg, J. (1999). La habitación vacante. Pretextos.

Norberg-Schulz, C. (1975). Existencia, espacio y arquitectura. Blume.

Riegl, A. (2007). El culto moderno a los monumentos, su carácter y sus orígenes. [1a ed. 1903]. Instituto Andaluz del Patrimonio Histórico.

Rodrigo Cámara, J. M., Díaz Iglesias, J. M., Fernández Cacho, S., Fernández Salinas, V., Hernández León, E., Quintero Morón, V., González Sancho, B. y López Martín E. (2012). Registro de paisajes de interés cultural de Andalucía. Criterios y metodología. Revista PH, (81), 6475

Rodríguez de Guzmán Sánchez, S. (Dir.) (2011). Avance de Plan Director del Conjunto Arqueológico de Itálica. Consejería de Cultura.

Rössler, M. (2002). Los paisajes culturales y la convención del patrimonio mundial cultural y natural: resultados de reuniones temáticas previas. En E. Mújica Barreda (Ed.), Paisajes culturales en los Andes (pp. 47-55). UNESCO.

Ruiz González, B. (Dir.) (2018). Plan Director del Conjunto Arqueológico Dólmenes de Antequera 2018-2025. Consejería de Cultura.

Ruiz González, B. (Dir.) (2010). Avance del Plan Director del Conjunto Arqueológico Dólmenes de Antequera (2011-2018). Consejería de Cultura.

Ruiz González, B. (Dir.) (1992). La Cartuja recuperada. Consejería de Cultura y Medio Ambiente.

Salmerón Escobar, P. (Coord.) (2004). Guía del paisaje cultural de la Ensenada de Bolonia, Cádiz. Avance. PH Cuadernos XVI. IAPH.

Salmerón Escobar, P. (2003). Paisaje y patrimonio cultural. En J. Fernández Lacomba, F. Roldán Castro y F. Zoido Naranjo (Coord.). Territorio y Patrimonio. Los Paisajes Andaluces. PH Cuadernos XV (pp.28-45). IAPH.

UNESCO (2016). Decision: 40 COM 8B.27. Antequera Dolmens Site. En Report of the Decisions adopted during the 40th session of the World Heritage Committee. Estambul (pp. 223-226). UNESCO. https://whc.unesco. org/archive/2016/whc16-40com-19-en.pdf
UNESCO (2012). Decision: 36 COM 8B.42. Rio de Janeiro, Carioca Landscapes between the Mountain and the Sea. En Decisions adopted by the World Heritage Committee at its 36th session. San Petersburgo (pp. 210-213). UNESCO. https://whc.unesco.org/archive/2012/whc1236com-19e.pdf

Vázquez Consuegra, G. (2020). Proyecto Básico y de Ejecución de Conservación y Adecuación para el nuevo Centro Cultural Atarazanas. Sevilla [proyecto inédito].

Venere, L. (2016). C'è una basilica paleocristiana in Puglia... in rete metallica. Artribune, 18 marzo 2016. https://www.artribune.com/attualita/2016/03/edoardotresoldi-basilica-paleocristiana-parco-archeologicosiponto-manfredonia/

Vivio, B.A. (2010). Franco Minissi. Musei e restauri. La trasparenza come valore. Gangemi Editori

Villalobos Gómez, A. (Coord.) (2021). Proyecto Museológico del Sitio de los Dólmenes de Antequera. Conjunto Arqueológico Dólmenes de Antequera.

Villalobos Gómez, A. (2020b). Principios para la tutela efectiva del patrimonio cultural. Revista PH, (101), 355 357.

Villalobos Gómez, A. (Coord.) (2020a). Sitio de los Dólmenes de Antequera. Intuición e intención en la obra de Javier Pérez González. Consejería de Cultura y Patrimonio Histórico.

Villalobos Gómez, A. (2017). El patrimonio es un umbral. Revista PH, (91), 164-166. 